\title{
Towards a Collaborative Network Paradigm for Emergency Services
}

\author{
Ovidiu Noran \\ Griffith University, Australiia \\ O.Noran@griffith.edu.au
}

\begin{abstract}
The rate and intensity of large scale disasters, natural and induced by humans via deforestation, carbon emissions, terrorism etc appears to be steadily increasing. At the same time, mankind appears to create increasingly complex systems to sustain its survival, quality of life and evolution. Ensuring that the people and their vital systems survive and cope with the natural disasters require proper preparation, response and recovery. Unfortunately, often the organisations put in place to deliver emergency services operate in a suboptimal way due to a plethora of factors, with a prominent role played by the lack of proper inter-operation and collaboration. This paper proposes the use of Collaborative Networks $(\mathrm{CN})$ concept in Emergency Services from an Enterprise Architecture (EA) perspective so as to take advantage of the wealth of knowledge accumulated in these domains. Following an introduction and a brief review of the state-of-the-art, the paper justifies the adoption of the $\mathrm{CN}$ paradigm and demonstrates the use of an EA approach previously tested for the $\mathrm{CN}$ domain, in the Emergency Services area.
\end{abstract}

Keywords: Disaster Management, Emergency Service, Enterprise Architecture, Collaborative Networks.

\section{Introduction}

Natural and man-made disasters appear to affect mankind in a more profound way than ever before. Globalisation, climate change and the increasing complexity of the systems ensuring our well-being and survival are a few crucial factors. Due to historical, administrative and geographical reasons, the organisations put in place to prepare for and assist the society in coping with the consequences of disaster events have evolved independently. The result is a heterogeneous collection of emergency services worldwide that often have significant difficulties in cooperating and interoperating in order to achieve a much needed synergy - as typically, handling disaster events require resources and capabilities beyond those of any individual organisation, whether local, state or national.

This paper proposes the use of a Collaborative Network (CN) approach in order to tackle some of the causes that cannot be addressed in the short amount of time available to respond to a disaster event. It argues that the interoperability-related issues must be addressed in advance and in a holistic manner - so that when a disaster event occurs, an efficient, collaborative task force can promptly be put together. 


\section{Collaborative Disaster Management - The Problems}

Emergency Services cooperation is often mandated by laws and frameworks at state, national and international level (e.g. [1-4]). However, simply mandating / coercing organisations into cooperation and using high-level generic frameworks does not bring true collaboration and / or interoperability. Consequences include extended response time, confusion / dispute as to who is in charge and when, difficulty in coordinating with other teams / systems due to incompatibilities in infrastructure and delays in validating and using the inevitable flood of information generated by a disaster event. For example, lack of agreement on the type and delivery format of warning messages may result in a lack of / delay in warnings or in the saturation of the intended audience with ambiguous/irrelevant warnings, leading to delayed or no preventive / evacuation action from the recipients and subsequent injury and life loss.

The typical approaches taken in trying to eliminate the above-mentioned problems have been either leaning towards centralisation / hierarchical command or towards the federalisation of emergency services. However, both alternatives have been criticised by authors and community response [5-7] because irrespective of the approach used, proper emergency response and cooperation has still not been achieved. The lack of preparedness and readiness appears to be caused by inadequate information and knowledge flow and quality between the participants [8, 9], lack of trust, organisational confusion and even misguided perception of competition. An alternative approach [10] proposes the use of military operations-inspired networkenabled capabilities as the backbone of disaster management. However, the authors recognise that this model works only as a component of the overall disaster management effort and could lead to excessive reliance on the infrastructure alone.

In finding a solution to this apparent conundrum, it must be recognised that true, prompt and efficient collaboration requires that organisational cultures, processes and resources of the participants must display the required preparedness [11]. This requires access to a plethora of interdisciplinary information and knowledge not always obvious or readily available to planners and disaster managers.

The above-described situation is significantly similar to that of commercial enterprises that need to cope with a global business environment requiring them to tackle projects beyond their own resources and knowledge. A typical response to this problem in the commercial domain is to set up or join Collaborative Networks (CNs) that act as 'breeding environments' for Virtual Organisations (VOs) that can be promptly created in order to bid for, win and complete projects requiring combined resources and know-how.

\section{Adopting a Collaborative Network Approach}

Calls to set up and improve cooperation through various collaborative artefacts are common in medical / emergency response reports, conferences and journals; unfortunately, the actual implementation of such recommendations is still limited.

Firstly, it is often difficult to even achieve stakeholder consensus (common image) on the present (AS-IS) situation, which hinders progress towards defining the optimal 
future (TO-BE) state of affairs. This is often accompanied by lack of agreement on an optimal roadmap to achieve the TO-BE caused by the various ways of 'getting things done' used by the participants, showing organisation, culture and even language gaps that result in a semantic interoperability barrier between the participants especially at middle and upper management level. Interoperability is paramount in the efficiency and survival of a $\mathrm{CN}$ - therefore it has been (and still is) extensively researched with aspects and dimensions identified and partial solutions proposed (e.g. [12,13] and many others). The difficult area of organisation and culture has also been tackled (e.g. [14]). This wealth of interoperability knowledge could be applied to the disaster management area under the $\mathrm{CN}$ paradigm.

Secondly, it appears that there is insufficient emphasis laid on the life cycle / life history aspect of the participant organisations, potential network and other relevant entities - including the disaster event(s). The interactions required to collaborate and interoperate must be considered in this context because all entities of interest are continuously evolving.

Adopting a $\mathrm{CN}$ approach for disaster management / emergency response organisations would result in multiple benefits. Technical (infrastructure) and syntactic interoperability aspects could be addressed in the beginning. However, importantly, the participants will also have the time to address the organisational / cultural interoperability aspect by getting to 'know each other' and thus build trust. Such human-related processes cannot be rushed and thus be successfully performed during the very limited set-up time or the operation of the disaster management task force (when all efforts and resources should be focused on the disaster event).

Enterprise Architecture (EA) is a change management approach bridging management and engineering best-practice. An EA perspective, by means of artefacts such as EA frameworks and an EA-based guiding methodology would complement the $\mathrm{CN}$ approach by facilitating common understanding among all stakeholders and formulating methods to set up / operate Disaster Management Collaborative Networks (DMCNs) covering all aspects relevant to the envisaged emergency response tasks.

\subsection{Specific Features of the Disaster Management Collaborative Network}

A DMCN would have some specific features that must be observed in order to achieve a working model. Thus, the time available for set-up of a Disaster Management VO (DMVO) is significantly shorter than that available for a project bidding process. In addition, the DMCN, its participants and the DMVO(s) produced would operate under tighter / legislated operational guidelines set by the relevant Governments and national / international frameworks. The commercial and competitive motivations of the typical $\mathrm{CN}$ participants would be transformed into the efficiency / interoperability motivation translated into lives and physical / intellectual property rescued. Thus, the classic decision processes to create / join / remain / leave the $\mathrm{CN}$ for most partners would be mandated from outside. Partial (reference) models should be abstracted out of the lessons learned from past disasters and used to speed up VO creation. They could be classified on type reflecting the location (e.g. flooding, tornadoes, wildfires, severe storms in Australia [15]) and customised to allow for specific intensity, duration, side-effects and consequences. 
The DMVO produced by the DMCN will not bid for projects, but rather be created for and assigned a specific one, namely the management of the disaster event. The management structure, communication infrastructure, and other organisational interoperability issues would have been sorted out in advance, ensuring prompt response (readiness) and thus addressing a frequent weakness of crisis management. Human-related aspects requiring time such as trust, culture, and recognition (the latter featuring prominently in volunteer-based organisations [16, 17]) could be more specifically tackled using the concept of an 'emergency services academy' with local (state based) branches providing training based on a unique interstate curriculum (see [18] for an early initiative).

The 'lead' (initiating) partner(s) within the DMCN could be the major government bureaus / agencies (e.g. Attorney General's Office, Federal Emergency Management Agency etc) while normal partners could be local and volunteer-based emergency services, contractors and other relevant third parties.

In conclusion, while significant challenges in the domains of $\mathrm{CN}$ [19] and Enterprise Interoperability $[14,20]$ remain, adopting the $\mathrm{CN}$ paradigm allows to use tested research results and more mature artefacts. The EA perspective adds the life cycle dimension and ensures a whole-system, consistent approach as further shown.

\section{Using Enterprise Architecture Artefacts in the Approach}

In order to test the concept, in this research we have selected a reference Architecture Framework (AF) obtained by generalising several other AFs and thus potentially expressive enough to contain all the elements necessary to express all relevant disaster management aspects using EA artefacts. This AF is GERAM (Generalised Enterprise Reference Architecture and Methodology), described in ISO 15704:2005 [21]. Among others, GERAM has been used in practice to guide EA projects [22], assess other enterprise AFs [23-25], build a structured repository of AF elements for a project management decision support system [26] and to define an Environmental Management approach for CNs [27]. The main component of the reference architecture of GERAM is a MF (called GERA) containing an extensive set of aspects including life cycle, management, organisation, human and decision, corresponding to various stakeholder concerns [28]. For more details on GERAM and GERA see [21].

Subsets of GERA MF can and have been used towards modelling formalisms used in the creation of life cycle-based business models. For example, Fig. 1 shows on the left hand side a high level representation of a disaster event, taking into account its relevant life cycle phases and possible relations to other events. The typical GERA 'Detailed Design' and 'Implementation' life cycle phases can designate particular features of the event(s) - e.g. earthquake time, epicentre and duration, or tsunami epicentre, spread, wave speed, height etc. Note that the detail of the diagram is limited as a high level business model and some irrelevant life cycle phases have been omitted (shown dashed in the figure). 

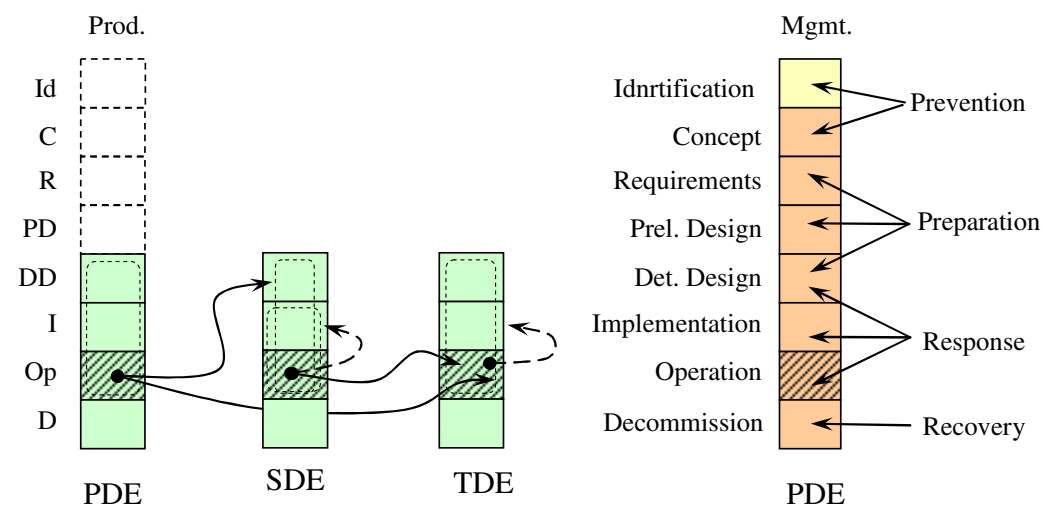

\section{Legend:}

PDE: Primary Disaster Event; SDE: Secondary Disaster Event; TDE: Tertiary ...

$\square$ : Production view $\square$ : Management view $\quad \rightarrow \rightarrow$ : Possible scenario

Fig. 1. Disaster event mapping and modelling using a GERA-based formalism

Using this kind of representation, it can be conveyed for example that a Primary Disaster Event (PDE) can trigger / influence other events (SDE, TDE). For example, an earthquake event (PDE) can trigger a tsunami (SDE) that can in turn trigger a partial nuclear meltdown (TDE). However PDE can also influence TDE directly. The diagram also shows that events can influence themselves (see arrows from Operation to Implementation within same entity) e.g. in the case of chain reactions etc. The right hand side of Fig. 1 shows how the life cycle phases of a disaster event can be mapped to typical disaster management activities [2]. Such graphical diagrams are useful in getting all the stakeholders to grasp a common understanding of disaster event aspects and help overcome the 'fuzzy' cultural / linguistic semantic interoperability barriers.

The paper argues that an EA approach can also provide an overarching life cyclebased approach in setting up and operating an emergency management $\mathrm{CN}$ aiming to produce emergency response VOs dealing with disaster events. In order to test this assumption, the author has used a 'method to build methods' applicable for specific types of EA tasks (projects), based on an original approach abiding by EA principles and tested in several case studies (e.g. [26, 29]). In a first step, the user is prompted to create a list containing entities of interest to the project in question, including project participants and target entities (organisations / systems being created). A second step comprises the creation of business models showing the relations between the previously identified entities in the context of their lifecycles, i.e. illustrating how entities influence each other during their life histories. The third and final step assists the user in inferring the set of project activities by reading and interpreting the previous representations for each life cycle phase of the project and other target entities. The resulting activities can be decomposed to a level deemed suitable for the intended audience. Due to its scope and to space limitations, the paper will cover only the first and second meta-methodology steps, focusing on the benefits of creating 
business models showing all relevant aspects of the participating entities. For comprehensive details on all meta-methodology steps see [29].

\section{Modelling the Emergency Services CN and VO}

Fig. 2 shows a possible business model of the creation and operation of a DMCN using a modelling formalism derived from the GERA MF. The level of detail in the figure has been purposely kept low (life cycles and management / production views only) so as to avoid confusion and to promote common understanding. If required, several diagrams may be added, focusing on various aspects - in which case attention must be paid to maintaining information consistency across models.

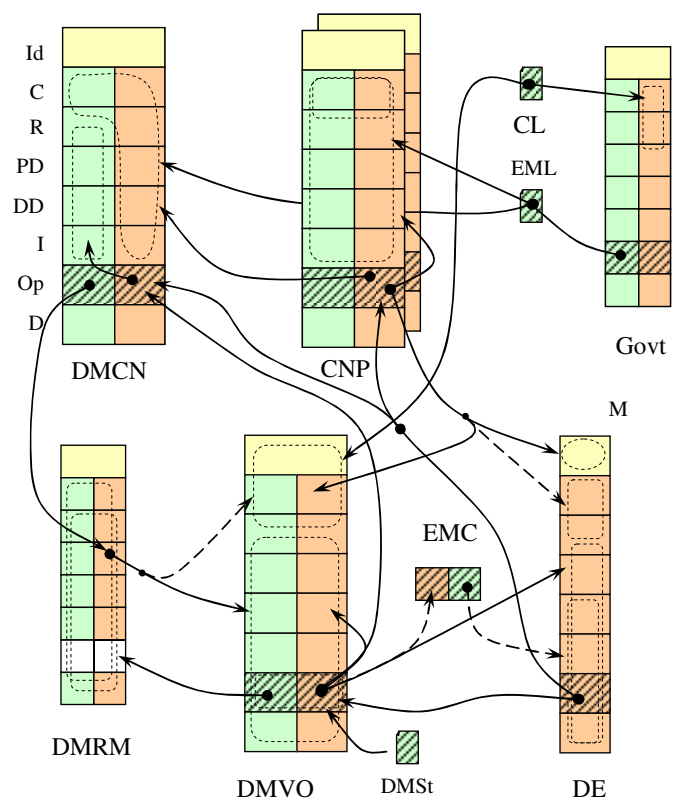

\section{Legend:}

CNP: Collaborative Network Participants;

DE: Disaster Event

DMCN: Disaster Mgmt CN

EML: Emrg. Mgmt Laws

DMSt: Disaster Mgmt Standards

EMC: Emrg. Mgmt Contractors;

DMRM:Disaster Mgmt Reference Model

DMVO: Disaster Mgmt VO

Gvt: Government

CL: Client (business/population)

$\dddot{Z Z Z}$ : Operation phase, Production \& Management

$\square$ : Operation Phase, Production

$\bullet-\rightarrow$ : Possible scenario

Life cycle phases: $\mathrm{Id}$ : Identification; $\mathrm{C}=$ concept; $\mathrm{R}=$ requirements, $\mathrm{PD}=$ preliminary design, $\mathrm{DD}=\mathrm{detailed}$ design, $\mathrm{I}=$ implementation, $\mathrm{O} \mathrm{p}=$ operation, $\mathrm{D}=$ decommissioning. Other aspects: $\mathrm{P}=$ Production / Service, $\mathrm{M}=$ management

Fig. 2. Life cycle-based business model of DMCN and DMVO creation / operation

The figure shows how a disaster event DE can be managed by a DMVO which is quickly put together by a DMCN, using suitable reference model(s) DMRM (if applicable). As can be seen, the DMVO would manage only the 'urgent' life cycle phases of DE (i.e. short-term preparation, response and recovery), while prevention and long-term preparation phases would be managed by the DMCN and possibly also by individual network members (CNP). Due to the importance of disaster management, significant parts of the CNP and DMCN life cycles are influenced directly by the local / federal Gvt and by national / international laws and treaties. 
The 'clients' (CL in Fig. 2) are businesses and population that are being assisted in case of DE and who provide feedback used to improve Gvt and DMVO in the future. Emergency management contractors EMC (or other third parties such as foreign rescue teams etc) contribute to DE management. While not necessarily part of the DMCN or DMVO, they must coordinate their efforts with the DMVO (arrow from DMVO operation to EMC management).

The arrow from DMVO's Management side of the Operation life cycle phase to some of its upper phases represents a very important 'partial redesign' capability, showing a need for the DMVO to be agile and adapt in real time in the face of rapidly changing conditions on the ground that are typical of some disaster events. Any major redesign will involve the DMCN, CNP, and Gvt and will be reflected in the subsequent DMVOs being created. Lessons learned in the operation of the DMVOs should be used towards building a repository of reference models (DMRM) that will help accelerate DMVO creation and thus improve emergency services readiness.

\section{Conclusions and Further Work}

Collaboration and interoperation are necessary and required in disaster management. Unfortunately, this often occurs in a sub-optimal manner due to the typical difficulties encountered when attempting to put together a heterogeneous task force in a very limited time. Lack of trust, different organisation / geographic cultures, diverse communication infrastructures and a limited or missing life cycle-based perspective of the disaster event(s) and of the participants' roles are decisive factors that can be addressed by prior preparation in a suitable environment. This paper has argued that emergency response organisations could significantly benefit by adopting a $\mathrm{CN}$ paradigm applied from an EA perspective to ensure a whole-system approach and has attempted to support this hypothesis by providing a high-level example involving EA artefacts. Further work is required to validate the proposed approach in several case studies involving various types of disaster events so as to triangulate the findings.

\section{References}

1. Government of South Australia. Emergency Management Act, 2004 (2004),

http: / / www. legislation.sa.gov.au/Lz/C/ EMERGENCY_MANAGEMENT_ACT2 004 .aspx (March 30, 2011)

2. Australian Government. Attorney's General's Office - Emergency Management in Australia (March 2011), http: / / www . ema . gov . au /

3. Federal Emergency Management Agency. National Response Framework (2011), http://www.fema.gov/pdf/emergency/nrf/about_nrf.pdf (March 30, 2011)

4. United Nations International Strategy for Disaster Reduction Secretariat (UNISDR). Hyogo Framework for Action 2005-2015: Building the resilience of nations and communities to disasters (March 2011), http://www.preventionweb.net/ 1037_hyogoframeworkforactionenglish.pdf

5. Clark, J.: Practical aspects of federalizing disaster response. Critical Care 10, 107-113 (2006) 
6. Wiese, C.R.: Organizing Homeland Security after Katrina: is adaptive management what's missing? Public Administration Review 66(3), 302-318 (2006)

7. 't Hart, P., et al.: The Politics of Crisis Management: Public Leadership under Pressure. Journal of Homeland Security and Emergency Management (2005)

8. Wickramasinghe, N., von Lubitz, D.K.J.E.: Knowledge Based Enterprises: Theories and Fundamentals. Journal of Homeland Security and Emergency Management 5 (2007)

9. Prizzia, R., Helfand, G.: Emergency preparedness and disaster management in Hawaii. Disaster Prevention and Management 10(3), 163-172 (2001)

10. von Lubitz, D.K.J.E., Beakley, J.E., Patricelli, F.: Disaster Management: The Structure, Function, and Significance of Network-Centric Operations. Journal of Homeland Security and Emergency Management 5(1), Art 42 (2008)

11. Kapucu, N., Arslan, T., Demiroz, F.: Collaborative emergency management and national emergency management network. Disaster Prev. and Management 19(4), 452-468 (2010)

12. Chituc, C.M., Azevedo, A.: Multi-perspective challenges on collaborative networks business environment. In: Camarinha-Matos, L., Afsarmanesh, H., Ortiz, A. (eds.) Collaborative Networks and Breeding Environments, pp. 25-32. Springer, Heidelberg (2005)

13. ATHENA State of the art of Enterprise Modelling Techniques and Technologies to Support Enterprise Interoperability. Deliv D.A1.1.1 (2004), http: / / www . athena-ip.org (cited March 30, 2011)

14. Whitman, L., Panetto, H.: The Missing Link: Culture and Language Barriers to Interoperability. Annual Reviews in Control 30(2), 233-241 (2006)

15. Ellis, S., Kanowski, P., Whelan, R.: National inquiry into bushfire mitigation and management. Commonwealth of Australia, Canberra (2004)

16. Esmond, J.: Report on The Attraction, Support and Retention of Emergency Management Volunteers (2011),

http: / /www. ema.gov. au/www/emaweb/emaweb. nsf / Page/Volunteers2 (cited March 2011)

17. McLennan, J.: Issues Facing Australian Volunteer-Based Emergency Services Organisations 2008 - 2010 (2008),

http: / /www. ema.gov. au/www/emaweb/emaweb.nsf/Page/Volunteers2 (March 30, 2011)

18. Queensland Government. Queensland Combined Emergency Services Academy, QCESA (2011),

http://www.safecom.sa.gov.au/site/emergency_management.jsp (March 2011)

19. Camarinha-Matos, L., et al.: Collaborative networked organizations - Concepts and practice in manufacturing enterprises. Computers and Industrial Engineering 57(1), 46-60 (2009)

20. Molina, A., et al.: Enterprise Integration and Networking: Challenges and Trends. Studies in Informatics and Control 16(4) (2007)

21. ISO/IEC, Annex C: GERAM, in ISO/IS 15704:2000/Amd1:2005: Industrial automation systems - Requirements for enterprise-reference architectures and methodologies (2005)

22. Bernus, P., Noran, O., Riedlinger, J.: Using the Globemen Reference Model for Virtual Enterprise Design in After Sales Service, in Globemen. In: Karvoinen, I., et al. (eds.) VTT Symposium, Helsinki / Finland, vol. 224, pp. 71-90 (2002)

23. Noran, O.: A Mapping of Individual Architecture Frameworks (GRAI, PERA, C4ISR, CIMOSA, Zachman, ARIS) onto GERAM. In: Bernus, P., Nemes, L., Schmidt, G. (eds.) Handbook of Enterprise Architecture, pp. 65-210. Springer, Heidelberg (2003) 
24. Noran, O.: An Analytical Mapping of the C4ISR Architecture Framework onto ISO15704 Annex A (GERAM). Computers in Industry 56(5), 407-427 (2005)

25. Saha, P.: A Synergistic Assessment of the Federal Enterprise Architecture Framework against GERAM (ISO15704:2000 Annex A). In: Saha, P. (ed.) Enterprise Systems Architecture in Practice, pp. 1-17. IDEA Group, Hershey (2007)

26. Noran, O.: Discovering and Modelling Enterprise Engineering Project Processes. In: Saha, P. (ed.) Enterprise Systems Architecture in Practice, pp. 39-61. IDEA Group, USA (2007)

27. Noran, O.: Towards an Environmental Management Approach for Collaborative Networks. In: Camarinha-Matos, L., Boucher, X., Afsarmanesh, H. (eds.) Collaborative Networks for a Sustainable World, pp. 17-24. Springer, Berlin (2010)

28. ISO/IEC, ISO/IEC 42010:2007: Recommended Practice for Architecture Description of Software-Intensive Systems (2007)

29. Noran, O.: A Meta-methodology for Collaborative Networked Organisations: Creating Directly Applicable Methods for Enterprise Engineering Projects (2008); VDM Verlag. Dr. Mueller 